\title{
Distance-near disparity esotropia: can we shrink the gap?
}

\begin{abstract}
Distance-near disparity esotropias are a group of heterogenous usually acquired strabismus disorders, where the angle of misalignment at near exceeds that at distance by 10 prism diopters or more, where the accurate correction of refractive errors and ambylopia are important early objectives. These aetiologically diverse entities respond non-uniformally to strabismus surgery and bifocals. The management challenge is one of 'shrinking' the disparity so that the affected individuals can develop and comfortably maintain binocular single vision and/or optimal alignment. Surgical procedures have continued to evolve but none of the current operative procedures are superior for all patients. Subclassifying this strabismus and highlighting publication data from more homogenous clinical series may assist with the optimisation of future management and treatment outcomes.
\end{abstract}

Eye (2015) 29, 208-213; doi:10.1038/eye.2014.277; published online 14 November 2014

\section{JP Burke, Department of} Ophthalmology, Royal Hallamshire Hospital Sheffield and the Children's Hospital Sheffield, Glossop Road, Sheffield, South Yorkshire S10 2JF, UK Tel: + 44 (0)114 2712223 ; Fax: + $44(0) 1142713747$ E mail: john.burke@ sth.nhs.uk

Received: 30 September 2014

Accepted: 14 October 2014 Published online: 14 November 2014

This paper was presented at the 44th Cambridge Ophthalmological Symposium, 'Ocular Motility' 4th and 5th September 2014

\section{Introduction}

Distance-near disparity (DND) esotropias were described by Donders in $1864 .{ }^{1} \mathrm{~A}$ review of the literature reveals numerous studies where outcome analysis has not been helped by the bundling together of patients with different clinicopathological entities. ${ }^{2}$ This lack of aetiological homogeneity in many published studies has complicated the development of a best practice framework that may arguably benefit from 'DND' subclassification so as to more accurately reflect and understand the sensory, motor, and psychosocial outcomes of each subgroup and develop and apply current surgical and non-surgical therapies optimally.

DND esotropia includes patients with high gradient $\mathrm{AC} / \mathrm{A}$ ratio (classical convergence excess) esotropias, those with normal AC/A ratios (the non-accommodative convergence excess group), those with a low $\mathrm{AC} / \mathrm{A}$ ratio with a remote near point of accommodation (NPA) known as hypoaccommodative convergence excess, ${ }^{3}$ partially accommodative distance esotropes with a high gradient AC/A ratio, patients with acquired or early-onset strabismus with a manifest distance esotropia with/without oblique dysfunction, and other miscellaneous cases.

Refractive or fully accommodative esotropia arguably represents an ideal 'best achievable' comparator for motor and sensory outcomes to the DND esodeviations. Ambylopia and anisometropia are common in the former at diagnosis. High grades of stereovision can be expected in up to $25 \%$ with fusion achievable in up to $75 \%$ of cases, especially in the many whose esotropia onset is after 2 years of age and spontaneous consecutive exotropia occurs in 5 to $6 \%$ of cases often after years of prior successful spectacle wear. ${ }^{4}$

Shrinking the gap

The successful management of the DND esotropias hinges on the optimal application of surgical and non-surgical therapies, where 'shrinking the gap' means achieving a residual angle of misalignment at distance and near that renders an individual asymptomatic or considerably improved with demonstrable stereovision and/or fusion in conjunction with a phoria or microtropia at all fixation distances while wearing an appropriate monofocal lens.

There should be a careful analysis of and emphasis on generating a preplanned target range that, if achieved, can be associated with a likelihood of successful ocular realignment. To this end, a knowledge of preoperative characteristics that might predict the likelihood of success/failure (eg, degree of hyperopia and refraction stability; magnitude of the measured gradient $\mathrm{AC} / \mathrm{A}$, the magnitude of the $\mathrm{DND}$, and the response to prism adaptation) are of value. ${ }^{5,6}$ 
Directing future research at ascertaining the extent to which these outcomes can be attained and improved upon in the various DND esotropia subgroups could lead to yet further progress.

\section{Classification}

DND esodeviations may be differentiated clinically on the basis of the NPA, the gradient AC/A ratio, the DND after fully correcting the underlying hypermetropia, the response to bifocals, and the magnitude of the misalignment at near and at distance (Table 1).

The most popular way of estimating the DND in North America is to compare the magnitudes of the distance and near heterophorias/heterotropias and consider a high $\mathrm{AC} / \mathrm{A}$ ratio as an alignment at $1 / 3 \mathrm{rd} \mathrm{m}$, that is, 10 prism diopters (PD) more convergent than that distance fixation. ${ }^{7}$ This method is popular and requires no additional clinical tasks or calculations. Havertape et $a l^{8}$ advised that the use of this difference to determine a high $\mathrm{AC} / \mathrm{A}$ ratios can be innacurate, as the $\mathrm{AC} / \mathrm{A}$ relationship was normal when measured by the gradient method in many patients with an abnormal DND. Likewise, von-Noorden et al, ${ }^{9}$ observed in a study designed to examine the role of bifocals in the treatment of accommodative esotropia, noted that some of these patients measured more overconvergence at near than at distance when simply using the distance-near comparison while their gradient $\mathrm{AC} / \mathrm{A}$ ratio was low and their response to bifocal therapy was poor.

It is standard to formally measure the $\mathrm{AC} / \mathrm{A}$ ratio in the United Kingdom using the gradient $\mathrm{AC} / \mathrm{A}$ measurement. It takes a little longer and has been reported as near eliminating proximal convergence from consideration. ${ }^{10,11}$

\section{Hypoaccommodative convergence excess}

Hypoaccommodative convergence excess is uncommon. Arnoldi, $^{12}$ in a review of 77 cases with a DND, reported a $1 \%$ incidence. Costenbader ${ }^{3}$ in 1958 described this group of strabismic patients characterised by a much reduced NPA and a low AC/A ratio. He postulated that they used excessive accommmodative effort to see clearly at near, and in doing so, exhibited an undesired excessive convergence of the eyes. It is managed with hypermetropic spectacle lenses. von Noorden and Jenkins ${ }^{13}$ re-evaluated this condition after fusing teenagers who had successfully worn bifocals for a mean duration of 4.3 years had an unexpectedly high frequency $(25 \%)$ of poor accommodative amplitudes and presbyopic symptoms. This study could not confirm whether the reduced accommodation occurred during or antedated bifocal therapy.
Fresina et $a^{14}$ in a prospective age-matched control study noted that deficient accommodation (slightly lower than average) was present in $10 / 28$ of their bifocal patients but in none of the controls. The NPA abnormalities predated bifocal wear and did not deteriorate while wearing bifocals over 4 years. This small prospective study supported the existence of a preexisting NPA deficit. Attempts to more routinely assess the NPA in children before and during bifocal therapy may prove clinically practical and have prognostic relevance, in that the excessive convergence at near is related to increased accommodative effort and a normal or near-normal $\mathrm{AC} / \mathrm{A}$, where prospective surgery may be potentially deleterious where there is a protracted requirement for reading or bifocal lenses, while 'bridging' the distance-near alignment gap. ${ }^{13}$

\section{Hypermetropic undercorrection}

Classically, the refractive error in high gradient AC/A ratio esotropia tends to be lower than that of fully accommodative esotropia where patients can be deliberately undercorrected by an amount gauged to the ease with which esodeviation is controlled. However, one needs to be mindful that in cases with a high gradient $\mathrm{AC} / \mathrm{A}$ minor degrees of hypermetropic undercorrection can result in classification and diagnostic errors and inaccurate diagnoses. Black ${ }^{15}$ observed that a significant minority of patients in his study with an abnormal distance-near relationship ( $\geq 10$ PD) ultimately did not need bifocal glasses following an increase of $0.5-0.75 \mathrm{D}$ in their monofocal lenses and normalisation of the DND. He reported that 19 of 51 patients had their DND normalised in this way, where the mean DND for this specific subgroup was 12 PD.

\section{Convergence excess esotropia with high gradient AC/A ratio: orthotropia/microtropia at distance}

This is the quintessential DND esodeviation that can be managed with bifocals, strabismus surgery, and, indeed, both where spontaneous deterioration or iatrogenic overcorrection, while visually immature, may result in a permanent loss of binocular single vision (BSV) including bifoveal fixation in a minority. ${ }^{5}$

Garretty ${ }^{16}$ reported an annual incidence in Leeds of six new cases per year (city population $=443000$ ). Kutschke and Keech ${ }^{17}$ measured the gradient $\mathrm{AC} / \mathrm{A}$ ratio in $62 \%$ of their DND cases. It was abnormally high in just $19 \%$, wheras Black ${ }^{15}$ reported that $22 \%$ of patients in their DND series responded to bifocals.

Leitch et al ${ }^{18}$ described the sensory outcomes of 31 children managed by primary surgery at a mean age of 5.7 (range 2.5-9.0) years. The majority (75\%) achieved 
Table 1 Classification of distance-near disparity esodeviations Hypoaccommodative convergence excess

Hypermetropic undercorrection

Convergence excess with high gradient $A C / A$ ratio Orthotropia/microtropia

Larger manifest angle

Distance-near disparity without high gradient $\mathrm{AC} / \mathrm{A}$ ratio

peripheral fusion/subnormal stereovision, 16\% achieved central or higher levels of stereovision, and $9 \%$ had no detectable fusion. There was a modest postsurgery improvement in sensory status in 67\%. Pratt-Johnson and Tillson ${ }^{19,20}$ analysed the outcomes in 80 bifocal users with a minimum 4-year follow-up, with similar sensory outcomes, where $76 \%$ achieved peripheral, $9 \%$ central, and a further $15 \%$ no detectable fusion. These data are approaching but inferior to the sensory outcomes described by Berk for Refractive Accommodative Esotropes. ${ }^{4}$

The maintenance of distance BSV during visual maturation is an important principle when attempting to improve on the sensory and motor outcomes from earlier studies, ${ }^{18-20}$ where earlier surgery is an increasingly appealing modern-day alternative to protracted bifocal wear because it potentially offers speedier alignment but risks added complications.

Current popular surgical strategies are augmented medial rectus recessions based on the magnitude of the near-angle, 5,21,22 preoperative prism adaptation for the near-angle ${ }^{23}$ and primary position sparing surgery. ${ }^{24}$ It is the surgeon's preference rather than clinical findings that in a large part determine the operation selected, where the aim is to reduce the DND so that any residual deviation at near and at distance lies within that individual's fusional amplitudes. Otherwise, the likelihood of persisting symptomatic undercorrections at near and symptomatic overcorrections at distance and near increase.

Relatively few publications have segregated DND patients into meaningful homogeneous subgroups. Arnoldi and Shainberg 5 analysed 37 such children who underwent augmented medial rectus recessions based on the larger near angle prospectively over 5 years. The age at primary surgery was $3.9 \pm 0.2$ years. There was an $80 \%$ success at 4 months defined as no documented loss of BSV and alignment within 8 PD of orthotropia at near and at distance without the need for bifocals that reduced to $30 \%$ at 5 years with the recurrence of an increasing gradient $\mathrm{AC} / \mathrm{A}$ within a year. There was a $43 \%$ near esotropia recurrence and a $27 \%$ consecutive distance exotropia rate and a mean of 2 operations per patient (range 1-5), where only 2 of the 19 patients who required $>1$ operation being eventually defined as a success.
The mean DND reduced from 23 to $12 \mathrm{PD}(P<0.01)$. The mean gradient $\mathrm{AC} / \mathrm{A}$ ratio reduced by 3.2 units. Binocular function was lost in $14 \%$ (including a child who had bifoveal fixation at baseline), remained stable in $59 \%$, and improved in $26 \%$. The successes differed statistically from failures at baseline in three ways. They had a larger distance deviation at outset $13 \pm 3$ vs $6 \pm 3$ PDs $(P<0.05)$, a significantly lower $\mathrm{AC} / \mathrm{A}$ ratio at outset $7.3 \pm 0.5$ vs $8.6 \pm 0.4(P<0.05)$, and a lower refractive error $1.56 \pm 0.7$ vs $4.16 \pm 0.6 \mathrm{D}(P<0.01)$.

Lueder and Norman ${ }^{23}$ reported retrospectively on 16 visually mature high $\mathrm{AC} / \mathrm{A}$ ratio esotropes who were bifocal dependent or indicated a preference to discontinue them. They underwent augmented bilateral medial rectus recessions based on prism adaptation for their near angle at a mean age of 8.3 years, and outcomes were defined as successful if they had a microtropia at near and at distance and maintained fusion without bifocals at a mean of 2.5 years.

There was an average of 1.3 (range 1-2) operations per patient and a mean increase of 11 PD in the surgical target angle during prism adaptation. Further surgery was more likely in the prism adaptation non-responder subgroup: $60 \%$ for consecutive exotropia and $40 \%$ for recurrent/persistent esotropia. Bifocals were eliminated in all with stable or improved binocular functions. Some $50 \%$ discarded spectacles entirely. Kushner ${ }^{25}$ cautioned disappointingly regarding the poor long-term ( $>5$ years) outcomes in overcorrected partially accommodative esotropes whose hyperopia was $>+2.50 \mathrm{D}$ when managed with postoperative spectacle manipulation.

Peterseim and Buckley ${ }^{24}$ performed bilateral medial rectus fadenoperations on 16 bifocal-dependent high gradient AC/A esotropes with a mean follow-up of 3 years and a mean age at surgery of 8.4 years. There were no reoperations or distance overcorrections but $18 \%$ were sufficiently undercorrected to intermittently require bifocals. Surgery resulted in a $71 \%$ reduction in the DND from 24 to $7 \mathrm{PD}$, a $61 \%$ reduction in mean gradient $\mathrm{AC} / \mathrm{A}$ (7.4-2.9) and improved binocular functions with 70\% achieving at least $400 \mathrm{~s}$ of stereovision compared with $44 \%$ preoperatively. They described a $70 \%$ and $36 \%$ (8-5.6 PD) reduction in the near and distance angles, respectively, whereas Akar et $a l^{26}$ described an 81 and $69 \%$ reduction in a partially accommodative esotropia subgroup.

All three procedures reduced the DND, the gradient $\mathrm{AC} / \mathrm{A}$, and the near and distance angles. There were no pulley fixation surgery ${ }^{27-29}$ reports for this homogeneous subgroup. Augmented medial rectus recessions are technically easier, whereas fadenoperations are challenging. On the other hand, isolated fadenoperations may be more prone to undercorrection but unlikely to overcorrect, whereas augmented surgery based on prism 
adaptation may by identifying prism builders reduce undercorrections but increase the long-term risks of overcorrection. ${ }^{23}$ The literature is unclear regarding the relative merits of sequential bifocal stabilisation followed by surgery near/after visual maturity compared with primary surgery at a younger age.

Preoperative prism adaptation should intuitively facilitate preoperative planning. It helps determine the smallest correctable target angle at near that is compatible with the achievement of comfortable BSV and the maximal change to the distance angle that can be tolerated by one's motor fusion, as prism adaptation in patients who have BSV is designed to 'stress test motor fusion at distance and near' so as to select a target range for surgery. Arnoldi and Shainberg's $\mathrm{s}^{5}$ supporting data described a potential protective effect from a larger underlying preoperative distance esodeviation. The most appropriate procedure could then be augmented medial rectus recessions, isolated faden operations, or combinations of both based on the relationship between the amount of misalignment that is deemed to require correction and the estimated fusional amplitudes, the DND, and the magnitude of the distance deviation (JP Burke, Personal Communication).

Bifocals are important to the management of children with a high AC/A ratio esotropia and are an especially practicable alternative to early surgery in the young visually immature child with distance BSV and limited cooperation. The further aim of bifocal wear is to promote the stabilisation or even expansion of fusional amplitudes so that patients can spontaneously discontinue the need for ongoing bifocal wear which currently occurs in a minority. ${ }^{9,30}$ They should also be considered as an important alternative next step in the stabilisation and management of initial surgical undercorrections.

\section{Partially accommodative distance esotropia with high gradient $\mathrm{AC} / \mathrm{A}$ ratio}

These DND patients will not routinely describe BSV at diagnosis, yet published motor alignment and sensory outcomes following surgical management are seemingly not dissimilar to the distance aligned/microtropic subgroup.

Akar $e a^{26}$ reported retrospectively on a group of 473 patients whose mean age at onset of strabismus was 2.9 years (range $0.5-14$ years), 365 of whom underwent bilateral fadenoperations in addition to medial rectus recessions where the preoperative mean distance angle was 44.9 PD (range 15-65), mean near angle 69.7 PD, and mean gradient $\mathrm{AC} / \mathrm{A}$ ratio 9.5 (range 5-12). The recessions were based on the measured distance angle using standard published ${ }^{31}$ surgical tables. Postoperative success was jointly defined as orthotropia or esotropia of $<10$ PD at near and at distance with available optical correction (reduced in some cases) and elimination of the DND.

Successful motor alignment occurred in $77 \%$, whereas $7 \%$ were exotropic and $16 \%$ esotropic. Some $75 \%$ of 473 patients had fusion and stereopsis better than $200 \mathrm{sec}$ of arc. The mean reduction in the near angle, distance angle, $\mathrm{DND}$, and $\mathrm{AC} / \mathrm{A}$ ratio were $88 \%, 86 \%$, $83 \%$, and $73 \%$, respectively, at 1 month postoperatively and these were not statistically different after a mean follow-up of 4.8 years. These sensory and motor findings were similar to the DND ortho/microtropic subgroup.

Kushner and Stolovich ${ }^{32}$ reported the effectiveness of augmented medial rectus recessions (without prism adaptation) based on the near angle for this patient subgroup in a frequently cited paper. He was careful to advise that the data could not be extrapolated to patients aligned at distance at diagnosis but requiring a bifocal to achieve near alignment. He reported a similar success rate at $82 \%$ (ie, 0-10 PD esotropia at near and at distance), and the discontinuation of bifocals in $95 \%$ of cases (a bifocal in one for a reduced NPA) without intentionally cutting the distance hypermetropia for alignment purposes. During the 15-year follow-up, 6 of 22 required additional surgery, 3 for esotropia/ exotropia and 3 for inferior oblique overaction. All reported sensory fusion, $18 \%$ with bifoveal fixation and a further $63 \%$ with stereovision that measured between 60 and $800 \mathrm{sec}$ of arc.

This literature suggests that cases with preoperative manifest acquired distance esotropia have the potential to demonstrate motor and sensory outcomes not dissimilar to those preoperative patients who are orthotropic/microtropic at distance.

\section{DND without high gradient $\mathrm{AC} / \mathrm{A}$ ratio}

Preoperative prism adaptation 33,34 was also adapted for the management of DND esotropia ${ }^{6,35}$ without a high gradient $\mathrm{AC} / \mathrm{A}$ ratio.

This subgroup is aetiologically ${ }^{36}$ and sensorially diverse and includes patients where a bifocal is incapable of reducing the near angle into a monofixation range. ${ }^{37}$ Accurate prevalence data for this subgroup is not readily attainable as many published studies did not attempt to segregate the various DND entities. Kutschke and $\mathrm{Keech}^{35}$ noted that the $\mathrm{AC} / \mathrm{A}$ ratio was normal in some $81 \%$ of study patients and is in excess of $75 \% 6,15$ in other publications.

Black $^{15}$ noted that the magnitude of the mean DND among the $22 \%$ of patients who responded to bifocal therapy was $23 \pm 7 \mathrm{PD}$ compared with $16 \pm 4 \mathrm{PD}$ in the bifocal non-responders, but $78 \%$ in majority of the 
groups. This heterogeneous study population included non-accommodative convergence excess patients, deteriorated fully accommodative or partially accommodative esotropes, cases of associated pattern strabismus, and even undercorrected hypermetropia in cases of fully and partially accommodative esotropia.

The patients wearing the optimal monofocal were managed surgically and a variety of techniques have been used with variable success. These again included augmented medial rectus recessions for the near deviation, preoperative prism adaptation based on the near-angle and pulley fixation or scleral posterior fixation sutures with/without medial rectus recessions.

\section{Conclusions}

In a recent survey of British and Irish practicing orthoptists $^{38}$ in relation to the $\mathrm{AC} / \mathrm{A}$ ratio measurement, it was noted that the $\mathrm{AC} / \mathrm{A}$ ratio is being widely used in the diagnosis of convergence excess esotropia $(97.26 \%$ of respondents), where the gradient method was the most popular among $93 \%$ of respondents and considered the most accurate by $92 \%$.

The current management of DND esodeviations has been formulated from many retrospective heterogeneous case series using data of variable rigour with follow-up time frames of quite variable duration. This renders the validity of some of the conclusions less robust and plausibly open to improvement. This is not an allencompassing bibliography review of DND esotropia, but has been designed to include published literature that describes more homogeneous patient subgroups.

It is arguably unrealistic to expect that the sensory and motor outcomes in this strabismus entity can ever exceed those of treated Refractive Accommodative Esotropia, which serves as a benchmark for further progress. In the past two decades there has not been a significant breakthrough either in terms of surgery or in neurophysical ways of enhancing motor fusion that could significantly advance management outcomes. Intuitively, some will argue, although by no means widely accepted, that surgical decision making has benefitted from the more time-consuming preoperative prism adaptation process. This process identifies prism builders, prism adaptation non-responders, and, in those minority of patients with established distance fusion before surgery, 'stress test' distance positive fusional amplitudes.

We know that a DND definition incorporates a spectrum of strabismic entities-mainly acquired but also congenital, with quite varied potentials for binocular cooperation, from negligible to high levels of stereovision, but yet they have generally tended to remain a literature-locked heterogeneous population.
Their subclassification and unbundling could contribute to consistently better clinical study designs that may then help improve outcomes in a more beneficial goaldirected manner. ${ }^{39}$

\section{Conflict of interest}

The author declares no conflict of interest.

\section{References}

1 Donders FC. On the Anomalies of Accommodation and Refraction of the Eye. The New Sydenham Society: London, UK, 1864.

2 Vivian AJ, Lyons CJ, Burke J. Controversy in the management of convergence excess esotropia. Br J Ophthalmol 2002; 86: 923-929.

3 Costenbader FD. Clinical course and management of Esotropia. In: Allen JH (ed). Strabismus Ophthalmic Symposium 11: CV Mosby-Year Book. CV Mosby: St Louis, MO, USA, 1958

4 Berk AT, Kocak N, Ellidokuz H. Refractive outcomes in Refractive Accommodative Esotropia. J AAPOS 2004; 8: 384-388.

5 Arnoldi K, Shainberg M. High AC/A ET: bifocals? surgery? or nothing at all? Am J Orthop 2005; 55: 62-74

6 Wygnanski-Jaffe T, Trotter J, Watts P, Kraft SP, Abdolell M. Preoperative prism adaptation in acquired esotropia with convergence excess. J AAPOS 2003; 7: 28-33.

7 Raab EL. Etiologic factors in Accommodative Esodeviation. Trans Am Ophthalmol Soc 1982; 80: 657-694.

8 Havertape SA, Cruz OA, Miyazaki EA. Comparison of methods for determining the $\mathrm{AC} / \mathrm{A}$ ratio in accommodative esotropia. J Pediatr Ophthalmol Strab 1999; 36: 178-183.

9 von Noorden GK, Morris J, Edelman P. Efficacy of bifocals in the treatment of accommodative esotropia. Am J Ophthalmol 1978; 85: 830-834.

10 Sloan LL, Sears ML, Jablonski MD. Convergenceaccommodation relationships. Arch Ophthalmol 1960; 63: 283-306.

11 Tait EJ. Accommodative convergence. Am J Ophthalmol 1951; 34: 1093-1107.

12 Arnoldi KA. Convergence excess characteristics and treatment. Am Orthop J 1999; 49: 37-413.

13 von Noorden GK, Jenkins RH. Accommodative amplitude in children wearing bifocals. In: Lennerstand G (ed). Update on Strabismus \& Pediatric Ophthalmology. CRC Press: London, UK, 1994, pp 201-204.

14 Fresina M, Schiavi C, Campos EC. Do bifocals reduce accommodative amplitude in convergence excess esotropia? Graefes Arch Clin Exp Ophthalmol 2010; 248: 1501-1515

15 Black BB. The influence of refractive error management on the natural history and treatment outcome of accommodative esotropia. Trans Am Ophthalmol Soc 2006; 104: 303-321.

16 Garretty T. Convergence excess esotropia, a proposed new classification and the effect of monocular occlusion. J Pediatr Ophthalmol Strabismus 2010; 47: 308-312.

17 Kutschke PJ, Keech RV. Surgical outcome after prism adaptation for esotropia with a distance-near disparity. J AAPOS 2001; 5: 189-192.

18 Leitch RJ, Burke JP, Strachan IM. Convergence excess esotropia treated surgically with fadenoperation and medial rectus muscle recessions. $\mathrm{Br}$ J Ophthalmol 1990; 74: 278-279. 
19 Pratt-Johnson JA, Tillson G. Sensory outcome with non-surgical management of esotropia with convergence excess (a high accommodative convergence/accommodation ratio). Can J Ophthalmol 1984; 19: 220-223.

20 Pratt-Johnson JA, Tillson G. The management of esotropia with high AC/A ratio (convergence excess). J Pediatr Ophthalmol Strabismus 1985; 22: 238-242.

21 Kushner BJ, Preslan MW, Morton GV. Treatment of partly accommodative esotropia with a high accommodative convergence-accommodation ratio. Arch Ophthalmol 1987; 105: 815-818.

22 O'Hara MA, Calhoun JH. Surgical correction of excess esotropia at near. J Pediatr Ophthalmol Strabismus 1990; 27: 120-123.

23 Lueder GT, Norman AA. Strabismus surgery for elimination of bifocals in accommodative esotropia. Am J Ophthalmol 2006; 142: 632-635.

24 Peterseim MMW, Buckley EG. Medial rectus fadenoperation for esotropia only at near fixation. J AAPOS 1997; 1: 129-133.

25 Kushner BJ. Partially accommodative esotropia; should you overcorrect and cut the plus? Arch Ophthalmol 1995; 113: 1530-1534

26 Akar S, Gokyigit B, sayin N, Demirok A, Yilmaz OF. Medial rectus faden operations with or without recession for partially accommodative esotropia associated with a high acciommodative convergence to accommodation ratio. Br J Ophthalmol 2013; 97: 83-87.

27 Clark RA, Ariyasu R, Demer JL. Medial rectus pulley posterior fixation is as effective as scleral posterior fixation for acquired esotropia with a high $\mathrm{AC} / \mathrm{A}$ ratio. Am J Ophthalmol 2004; 137: 1026-1033.

28 Mitchell L, Kowal L. Medial rectus pulley (posterior fixation) sutures in accommodative and partially accommodative esotropia with convergence excess. J AAPOS 2012; 16: 125-130.
29 Wabulembo G, Demer JL. Longterm outcome of medial rectus recession and pulley posterior fixation in esotropia with high AC/A ratios. Strabismus 2012; 20: 115-120.

30 Vivian A, Kousoulides L, Fells P, Lee JP. Posterior fixation sutures (faden operation) for the management of convergence excess esotropia. In: Lennerstrand G (ed). Update on Strabismus and Paediatric Pphthalmology. CRC Press: Boca Raton, FL, USA, 1994, pp 395-398.

31 Rosenbaum AL, Santiago AP. Clinical Strabismus Management: Principles and Surgical Techniques. WB Saunders: Philadelphia, PA, USA, 1999.

32 Kushner BJ, Stolovich C. Fifteen year outcome of surgery for near angle in patients with accommodative esotropia and a high AC/A ratio. Arch Ophthalmol 2001; 119: 1150-1153.

33 Repka MX, Connett JE, Scott WE. Prism Adaptation Study Research Group. The one year surgical outcome after prism adaptation for the management of acquired esotropia. Ophthalmology 1996; 103: 992-998.

34 Prism Adaptation Study Research Group. Efficacy of prism adaptation in the surgical management of acquired esotropia. Arch Ophthalmol 1990; 108: 1248-1256.

35 Kutschke PJ, Keech RV. Surgical outcome after prism adaptation for esotropia with a distance-near disparity. J AAPOS 2001; 5: 189-192.

36 Parks MM. Abnormal accommodative convergence in squint. Arch Ophthalmol 1958; 59: 364-380.

37 von Noorden GK, Avilla CW. Nonaccommodative convergence excess. Am J Ophthalmol 1986; 101: 70-73.

38 Murray C, Newsham D. Survey of British and Irish orthoptists in the measurement of the $\mathrm{AC} / \mathrm{A}$ ratio. Br Ir Orthopt J 2014; 11: 20-27.

39 Ehrenberg M, Nihalani BR, Melvin P, Cain CE, Hunter DG, Dagi LR. Goal-determined metrics to assess outcomes of esotropia surgery. J AAPOS 2014; 18: 211-216. 